\title{
La vinculación comunitaria desde la mirada de URACCAN: Experiencia en la elaboración del esta- tuto de la comunidad de Sumubila, RACCN
}

\author{
The community linkage from URACCAN perspective: An Experience in the elaboration of the Sumubila \\ Community Bylaw, RACCN
}

Sandra Carolina Rojas Hooker ${ }^{1}$

\section{Resumen}

Este ensayo presenta los aportes de la URACCAN como Universidad Comunitaria Intercultural en los procesos de vinculación Universidad-Comunidad en la elaboración participativa del "Estatuto de la comunidad multiétnica de Sumubila, Territorio de Tasba Pri, Puerto Cabezas, RACCN” como documento de referencia para la consolidación de la gobernanza comunal, reglamentar los derechos y obligaciones de los comunitarios, y, sobre todo, establecer algunas pautas para el uso sostenible de los recursos naturales, entre otros aspectos para alcanzar el buen vivir de la comunidad de Sumubila. En este proceso de acompañamiento, la comunicación horizontal y el diálogo abierto entre las partes fue fundamental para la producción de conocimientos. Nos permitió transitar por diferentes experiencias en el ámbito comunal-territorial, con una población multiétnica e intercultural como un modo de abrirnos paso a paso en la creación, recreación de conocimientos saberes y prácticas que fortalecen la estructura tradicional ancestral, elaborados con/ desde y para la comunidad de manera participativa y colaborativa.

Palabras clave: autonomía; diálogo de saberes; interculturalidad; estatuto comunal; vinculación universidad-comunidad.

\begin{abstract}
This essay presents the contributions of URACCAN as an Intercultural Community University in the University-Community linking processes for the participatory elaboration of the "Sumubila Multi-ethnic Community Bylaw, Tasba Pri Territory, Puerto Cabezas, RACCN" as a reference document for the consolidation of communal governance, regulating the rights and obligations of community members, and, above all, establishing some guidelines for the sustainable use of natural resources, among other aspects to achieve the good living of Sumubila community. In this accompaniment | process, horizontal communication and open dialogue between the parties were essential for the production of knowledge. It allowed us to travel through different experiences in the communal-territorial sphere, with a multiethnic and intercultural population as a way of opening ourselves step by step in the creation, recreation of knowledge and practices that strengthen the traditional ancestral structure, elaborated with / from and for the community in a participatory and collaborative way.
\end{abstract}

Keywords: Autonomy; Knowings Dialogue; Interculturality; Community Bylaw; University - Community Linkage.

\footnotetext{
1 Máster en Estudios Indígenas. Directora del Instituto de Estudios y Promoción de la Autonomía de la Universidad de las Regiones Autónomas de la Costa Caribe Nicaragüense - Recinto Universitario Bilwi. Email: srojashooker@gmail.com, ORCID: https://orcid.org/0000-0002-7467-2366 


\section{Introducción}

En la actualidad resulta oportuno plantear la relación entre la universidad y la sociedad, así se puede evidenciar que la universidad tiene una función social, que incide y transforma su entorno. La universidad debe contribuir a la solución de los problemas críticos que aquejan a la sociedad y ellos deben percibirse a través de la identificación de necesidades sociales, económicas y políticas que son poco satisfechas. Mediante la articulación de la docencia, la investigación y la extensión como componentes básicos de una institución de educación superior se hace posible materializar estas demandas. Las universidades y otros tipos de Instituciones Interculturales de Educación Superior (IIES) se caracterizan por integrar los saberes, modos de producción de conocimientos y modos de aprendizajes de varias tradiciones culturales. (Mato 2016:79).

En el modelo de educación superior intercultural las prácticas de extensión universitaria se les llaman de vinculación comunitaria. La razón de esta singularidad se debe a que se piensa que la vinculación establece una relación recíproca entre la comunidad y la universidad, de tal suerte que la comunidad se nutre por los saberes universitarios y la universidad por los saberes comunitarios (Casillas y Santini, 2006).

El presente documento analiza el proceso de vinculación comunitaria y diálogo de saberes con autoridades comunales. Presenta la experiencia del Instituto de Estudios y Promoción de Autonomía (IEPA) de URACCAN en la elaboración del estatuto de la comunidad de Sumubila, dando a conocer los procesos desarrollados y los aprendizajes obtenidos.

\section{Desarrollo}

\section{Nuestra Universidad}

En las últimas tres décadas, las luchas e iniciativas de los pueblos indígenas en varios países latinoamericanos han dado lugar a la creación de universidades y otros tipos de instituciones de educación superior. Algunas de ellas han sido establecidas y son gestionadas por organizaciones de estos pueblos, otras han sido creadas por agencias gubernamentales (Mato 2016:72).

La Universidad de las Regiones Autónomas de la Costa Caribe Nicaragüense (URACCAN) nace en 1994. Es un proyecto institucional de universidad propia, que responde a las aspiraciones históricas de los pueblos y comunidades indígenas, afrodescendientes y mestizos del Caribe por gozar de un modelo de desarrollo construido desde las bases socioculturales. Su filosofía institucional está orientada a revitalizar, promover y fortalecer el ejercicio de derechos colectivos, identidades, cosmovisiones, espiritualidades, formas de organización, culturas, sistemas de conocimientos y prácticas. Esto se da al mismo tiempo de evitar la fuga de talentos humanos, fortalecer la Autonomía Regional Multiétnica y el Subsistema Educativo Autonómico Regional (URACCAN 2015: 16 -17).

La URACCAN representa entonces un modelo de Universidad Comunitaria Intercultural definido por ejes, principios y valores, configurados en nuestro paradigma educativo, los cuales se operativizan en cada uno de los procesos, programas, proyectos, actividades y acciones que realiza. Su quehacer está determinada por cincos funciones sustantivas: i) Comunidades de Aprendizajes, ii) Creación y Recreación de Conocimientos Saberes y Prácticas, iii) Acompañamiento e Incidencia Social Comunitaria, i) Gestión y Comunicación Intercultural, v) Cooperación Solidaridad y Complementariedad.

El plan estratégico participativo de la URACCAN (PEI 2015-2019) define el acompañamiento e incidencia social y comunitaria como un proceso de diálogo de saberes implementado por la universidad con las comunidades y actores sociales; encaminados al ordenamiento de desarrollo regional y fortalecimiento de 
los sistemas autonómicos y la promoción del patrimonio cultural desde un enfoque de equidad de género, para la construcción colectiva del buen vivir de los pueblos, el respeto, equilibrio y armonía con la Madre Tierra (URACCAN, 2005:22).

La política de acompañamiento comunitario intercultural por su parte la define como un proceso colaborativo, participativo, concertado e inclusivo, sustentado en el diálogo de saberes y haceres, mediante la articulación entre universidad-comunidad- sociedad, que busca transformaciones estructurales, sociales, económicas, políticas, culturales y ambientales de los sistemas que sustentan las desigualdades en torno a la autonomía y autodeterminación de los pueblos, en pro del desarrollo con identidad y el ejercicio efectivo de las ciudadanías interculturales de género, para la construcción colectiva del buen vivir de los pueblos, el respeto, equilibrio y armonía con la Madre Tierra (URACCAN 2017:22).

Para operativizar la política de acompañamiento comunitario intercultural se establecen las siguientes líneas de estratégicas que dan salida a los diferentes ámbitos temáticos que trabaja la universidad a través de proceso formativo, acompañamiento a la formulación de planes de vida, movilización social, fortalecimiento de las organizaciones de base, la participación efectiva en redes, campañas sociales, investigación acción participativa, y que se explicitarán en el modelo de acompañamiento comunitario intercultural, en articulación con las diferentes funciones y estamento de la universidad.

Esta responsabilidad social de acompañar a los pueblos en su fortalecimiento, está impregnada por tres elementos que se constituyen en los ejes filosóficos y epistemológicos del quehacer institucional:

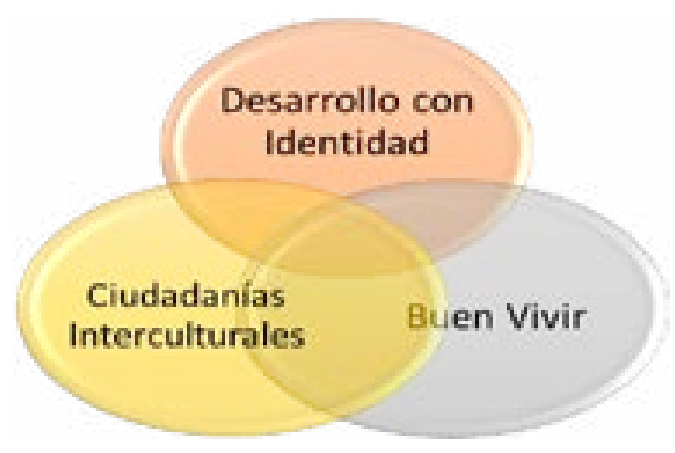

Figura 1: Ejes filosóficos y epistemológicos del quehacer institucional

En la práctica, la gestión estratégica del proceso de acompañamiento se realiza desde la Rectoría a través de los cuatro Institutos y dos Centros de Investigación: Instituto de Medicina Tradicional y Desarrollo Comunitario (IMTRADEC); Instituto de Recursos Naturales, Medio Ambiente y Desarrollo Sostenible (IREMADES); Instituto de Promoción e Investigación Lingüística y Cultural (IPILC); Instituto de Estudios y Promoción de la Autonomía (IEPA); Instituto de Comunicación Intercultural (ICI); Centro de Estudios e Información de la Mujer Multiétnica (CEIMM); Centro de Información Socio Ambiental (CISA) en articulación con la direcciones de áreas sustantivas de la universidad. 
Las instancias especializadas temáticamente investigan y acompañan al liderazgo comunitario, territorial y regional en la elaboración de propuestas dirigidas a mejorar las condiciones de vida de los pueblos; vinculados a la docencia especializada y a la asesoría técnica en aspectos relativos a sus competencias; así como a los procesos de acompañamiento consignados en la política que realizamos, con la participación de estudiantes, la academia universitaria y asociados cuando requiera el caso.

Para la URACCAN las comunidades representan laboratorios naturales invaluables con aprendizajes activos para actividades prácticas de sistematización, investigación e innovación, la formación integral del estudiantado como ser humano que comparte, valora y respeta los conocimientos, prácticas y cosmovisiones indígenas, afrodescendientes y mestizas (URACCAN, 2017:26). Esta práctica de vinculación se constituye como un aprendizaje, en la medida que tanto los miembros de la comunidad como el equipo de acompañamiento y los estudiantes, aprendemos colectivamente de estos procesos.

\section{Vinculación comunitaria y diálogo de saberes}

La generación de conocimientos en la interacción entre pueblos indígenas, afrodescendientes, mestizos y otras comunidades étnicas y la universidad es un proceso permanente de acompañamiento de doble vía. Tiene como finalidad la construcción de planes, proyectos, estrategias, acorde a los modos de vida de las comunidades y pueblos para el buen vivir, en correspondencia con el modelo de universidad comunitaria intercultural, tal a como mandata nuestro proyecto educativo institucional.

Las experiencias que se desarrollan desde la educación superior con/para/de pueblos indígenas y afrodescendientes nos ofrecen oportunidades de aprendizaje transformadoras a partir del dialogo de saberes entendida como un proceso comunicativo en el cual se ponen en interacción dos lógicas diferentes: la del saber cotidiano local y la del conocimiento científico-técnico, con una clara intensión de comprenderse, apreciarse y apoyarse mutuamente. Esta se encuentra fundamentada en el respeto y en la práctica de relaciones horizontales y democráticas.

Para URACCAN el diálogo de saberes y haceres es:

Una práctica o proceso organizado de análisis que propicia la creación, recreación, diseminación e intercambio de conocimientos, sentires, sabidurías, saberes y haceres, en la búsqueda permanente del buen vivir personal, familiar y comunitario, desde la armonía entre el conocimiento propio y el conocimiento occidental, con o sin la presencia de la universidad, donde prevalece el valor de la palabra y la escucha respetuosa para alcanzar la comprensión común y la plenitud de la vida (URACCAN 2017: 14 -15).

Implica romper con la idea de que hay verdades absolutas y con la visión de que solo el saber científico es verdadero y que los saberes populares son falsos; exige esta visión tener humildad, entender que: no eres dueña/o de la verdad, el conocimiento está allí en el mundo que te rodea y debes acercarte a él desde lo que tú conoces, para exponer dicho conocimiento, entrar en contacto con otros saberes y tener la disposicion de cambiar lo que se cree saber sobre los nuevos planteamientos que surjan de la interaccion de tus saberes con los de las personas con quienes compartes. (Bernal 2014:40).

Por tanto, el diálogo ocurre cuando se crea un escenario propicio en donde cada uno entenderá al otro en sus propios términos y desde saberes distintos, pero ambos dispuestos a aprender mutuamente uno del otro. En URACCAN la vinculación con los pueblos y comunidades se materializa a través de procesos de acompañamiento comunitario intercultural con y para los pueblos indígenas, afrodescendientes, mestizos y otras comunidades étnicas en donde la colaboración intercultural juega un papel preponderante para el desarrollo de las funciones sustanciales de la universidad. Estos diálogos y formas de colaboración deben 
ser honestos y respetuosos, de interés reciproco, que partan de reconocer que hay diversidad de contextos y de prácticas intelectuales de saberes.

Mato (2008:113) señala que, los problemas más difíciles de resolver para desarrollar formas y experiencias específicas de colaboración intercultural son los de "traducción". Con este término, Mato no alude tan sólo a los problemas de traducción de palabras e ideas de una lengua a otra, sino a los de visiones del mundo, sensibilidades y sentido, que son problemas de comunicación intercultural, sobre las cuales debemos trabajar con cuidado en cada caso y contexto. Reitera que la colaboración intercultural en la producción de conocimientos es imprescindible y ventajosa para todas las partes inmersas en el proceso.

\section{Construcción participativa del estatuto de la Comunidad de Sumubila}

La Constitución Política de Nicaragua de 1987 reconoce de manera expresa la existencia de comunidades indígenas y étnicas de la Costa Atlántica de Nicaragua. Por ende, la naturaleza multiétnica y pluricultural de la nación. A la vez reconoce los derechos de las comunidades de la Costa Atlántica al uso, goce y disfrute de las aguas, bosques y tierras comunales, preservar sus lenguas, religiones, arte y cultura; goce, uso y disfrute de las aguas, bosques, la creación de programas que coadyuven a su desarrollo y el derecho a las formas de organización social y de gobierno.

La Asamblea Nacional en ese mismo año promulga la Ley No. 28, Estatuto de Autonomía, creando el régimen legal de autonomía para las Regiones de la Costa Caribe y su posterior reglamento a través del Decreto A.N. No. 3584 de octubre del 2003.

En enero del 2003 entra en vigencia la Ley No. 445 que organiza el régimen de propiedad comunal de los pueblos indígenas y afrodescendientes como un mecanismo para formalizar la seguridad jurídica en la tenencia de la tierra. Se autoriza el establecimiento de un marco institucional para la demarcación y titulación de territorios y los procedimientos para titulación, así como su participación en las decisiones referentes a la tierra, territorio y recursos naturales.

Desde el 2007 a noviembre 2016 se han titulado 23 territorios: 16 en la Costa Caribe Norte, tres en la zona de régimen especial (Jinotega) y cuatro en la Costa Caribe Sur que corresponden a 304 comunidades. Uno de esos territorios es Tasba Pri, ubicado en el Municipio de Puerto Cabezas, RACCN integrado por diez comunidades: Kukalaya, Sahsa, Altamira, Kuakuil II, Naranjal, San Miguel, Nazareth, Columbus, Akawasito y Sumubila.

En virtud del memorándum de entendimiento suscrito entre el Instituto de Investigación y Gestión Social (INGES) y la Universidad URACCAN para la ejecución de cursos de educación continua se da el primer contacto con las autoridades territoriales de Tasba Pri y autoridades comunales de Sumubila. Aprovechando ese espacio, en el ejercicio de la autonomía comunal solicitan el acompañamiento de la URACCAN a través del Instituto de Estudios y Promoción de la Autonomía (IEPA) para la construcción participativa de su estatuto comunal.

Para las autoridades ancestrales, el estatuto es la ley de la comunidad. Representa el conjunto de acuerdos escritos de manera ordenada en un documento sobre la vida en comunidad, los derechos y obligaciones de los comunitarios, el aprovechamiento racional y sostenible de los recursos naturales y sobre todo, los mecanismos para el mantenimiento de la armonía y paz en la comunidad.

Se trabajaron dos niveles de articulación: Una articulación interna para lograr la optimización de los recursos financieros, recursos materiales y talentos humanos para trabajar en este proceso y una articulación 
externa con las autoridades comunales, con miras a establecer los mecanismos a utilizar, para la buena marcha del presente trabajo. Se conformó un equipo interdisciplinario integrado por un equipo técnico de URACCAN y representantes de la comunidad quienes de manera conjunta definimos la metodología a utilizar, lugar, duración de los encuentros y participantes.

El primer taller participativo se desarrolló en Sumubila por un período de tres días con la participación de 35 líderes y líderes previamente seleccionados a saber: el síndico, wihta o juez comunal, ancianos, coordinadores de sectores, líderes religiosos, de salud, educación, parteras, jóvenes, hombres y mujeres de la comunidad. Su finalidad: recopilar insumos para la construcción colectiva del estatuto de Sumubila, Tasba Pri, Municipio de Puerto Cabezas.

Metodológicamente se auxilió de las técnicas de grupos focales combinadas con trabajos de grupos temáticos. Se elaboraron guías acerca de distintos temas que son a la vez los ejes del proceso y que constituirían los elementos esenciales del estatuto de la comunidad. Para obtener los insumos, de manera colectiva se discutió y dio salida a las siguientes interrogantes: ¿Cómo era en el pasado?, ¿Cómo es el presente? y ¿Cómo quiere que sea en el futuro? focalizándola sobre la experiencia vivida, cuyos elementos son los cuatro "existenciales" básicos para los análisis antes mencionados: el espacio vivido (espacialidad), el cuerpo vivido (corporeidad), el tiempo vivido (temporalidad) y las relaciones humanas vividas (relacionabilidad o comunalidad).

Para los trabajos por grupos temáticos, se dividieron a los participantes por área de conocimiento para obtener insumos para construir los capítulos y artículos del estatuto.

De manera conjunta identificamos algunas ventajas de tener un estatuto comunal, a saber:

1. Ordena las funciones que les toca realizar a las autoridades ancestrales y las personas que viven en la comunidad.

2. Permite establecer mecanismos de protección de las tierras y de los recursos naturales definidos y creados por la propia comunidad.

3. Establece reglas para rescatar el sistema normativo comunitario, la vivencia comunitaria y su relación con la tierra y el territorio.

4. Permite describir las reglas para el uso y aprovechamiento sostenible de los recursos naturales existentes en la comunidad.

5. Fortalece el sistema de organización tradicional ancestral existente en la comunidad y su relación con las autoridades estatales.

6. Es un medio para reconocer la importancia de la mujer en las comunidades y garantizar su participación en los espacios de toma de decisión.

7. Establece los procedimientos para solucionar los conflictos o desavenencias que suscitan a lo interno de la comunidad y las formas de articulación con el sistema estatal en caso de ser necesario.

En el segundo y tercer día iniciamos con el análisis y discusión de aspectos medulares que debe contener el estatuto comunal. Se vio la necesidad de tener información de primera mano sobre la organización social de la comunidad, administración de justicia, administración de recursos naturales, administración 
de recursos financieros y sistemas de elección de autoridades y líderes comunitarios. En una matriz de resultados, se trabajó según los descriptores antes señalados y cuál era la situación en la comunidad antes, ahora y como se visualizan en el futuro para poder incorporarlos en el documento, siendo estos: conflictos políticos vinculados al proceso de elección de autoridades comunales, interétnicos, tenencia de la propiedad y violencia doméstica, aspectos a los cuales se brindó especial énfasis buscando una mejor manera de abordar dicha problemática respetando la cultura y normativa comunitaria.

Estos y otros aspectos fueron analizados y trabajados en sesiones posteriores con el equipo interdisciplinario para asegurar los aspectos de fondo y forma que debe contener el estatuto, contando en estos momentos con un borrador de la normativa comunitaria, distribuida en doce capítulos: I. Disposiciones generales, II. Principios y valores fundamentales, III. Derechos y deberes de los comunitarios; IV. Derechos y deberes de las personas con título de posesión en tierras comunales; V. Del patrimonio de la comunidad; VI. División administrativa; VII. Estructura y funciones del gobierno comunal; VIII. De la administración de justicia; IX. Del presupuesto de la comunidad. X. Elección, renuncia y destitución de las autoridades comunales, VI. Reforma de los estatutos y XII. Disposiciones finales.

La información se construyó de manera articulada con los comunitarios. Mediante el diálogo de saberes y haceres se logró conocer el contexto, analizar y plasmar en el documento los aspectos fundamentales de la vida comunitaria basadas en las necesidades de la comunidad, que nos permitió establecer pautas para alcanzar el buen vivir de la comunidad de Sumubila.

Se establecieron las coordinaciones con las autoridades ancestrales para la realización de la asamblea comunal con miras a presentar y discutir el contenido del estatuto artículo por artículo. Dicha Asamblea, se llevó a cabo el 24 de junio del presente año, en donde se dio a conocer el presente estatuto e incorporo las observaciones hechas por los comunitarios para poder contar con un documento terminado procediendo a la lectura y aprobación del Estatuto por parte de la Asamblea Comunal como máxima autoridad de la comunidad, de conformidad con el artículo 4, de la ley 445, ley de régimen de propiedad comunal.

\section{Logros}

A 24 años de fundada la URACCAN y de haberse institucionalizado los procesos de acompañamiento comunitario intercultural hemos logrado un reconocimiento, y aceptación por parte de los diferentes pueblos.

Lo anterior, nos permitió realizar programas, proyectos y procesos en/con/desde y para los pueblos y comunidades de la Costa Caribe Nicaragüense, tal es el caso de la formulación del estatuto de gobernanza territorial. Esta experiencia se desarrolla de manera articulada con las autoridades comunales de Sumubila mediante la recolección y sistematización de las normas tradicionales de dicha comunidad, que será parte integrante del conjunto de normas vigentes para los diferentes procesos que se desarrollan con/desde/ para la comunidad.

\section{Lecciones aprendidas}

La apropiación comunal de esta herramienta ha sido clave para el desarrollo de este proceso, logrando la participación de la comunidad en sus diferentes fases. Parte del éxito de la experiencia se debió a la articulación con los diferentes actores tanto de URACCAN como de la comunidad para la construcción colectiva de la normativa. Asi como la estimulación de la participación de cada uno de ellos, sobre todo los jóvenes y las mujeres, asegurando el respeto y la aplicación de la consulta y consenso como aspectos fundamentales del consentimiento libre, previo e informado estatuido en el convenio 169 de la OIT y otras normativas internacionales como nacionales. 
Si bien, la información contenida en el estatuto tiene que ver con el contexto actual, es menester reconocer la importancia y significado que tiene para los comunitarios de Sumubila el contar con un documento construido en/con/desde y para su comunidad, en donde la información y las opiniones de las partes fueron tomadas en cuenta.

El debate sobre la gobernanza y los recursos naturales son de suma actualidad e involucra el sustento jurídico e institucional de la comunidad. La construcción participativa y colaborativa de este instrumento jurídico genera modos de comprensión compartidos entre los actores involucrados, respecto a las principales amenazas que atentan contra su territorio, así como los retos y las oportunidades para su fortalecimiento $y$, por ende, la coordinación que debe existir entre los cuatro niveles de gobierno (Comunal, Territorial, Municipal, Regional).

Esta práctica de vinculación comunitaria en la construcción conjunta de la normativa comunitaria partió de las necesidades identificadas y que mediante el diálogo de saberes y la colaboración intercultural fue posible orientar los esfuerzos y las acciones para satisfacer las necesidades de la comunidad.

En el marco de este proceso, se firmó convenio de colaboración con dicha comunidad para continuar con los procesos de acompañamiento con los cuatro niveles de gobierno (comunal, territorial, municipal y regional) para la gestión y fortalecimiento de capacidades locales.

\section{Retos}

Uno de los mayores retos de esta experiencia es la implementación efectiva de esta normativa por parte de los comunitarios una vez aprobada la misma en Asamblea Comunal.

Cuerpo de leyes que deberá formar parte integral de las normativas que protegen los derechos colectivos, en consonancia con las leyes nacionales e internacionales.

Lo anterior permitirá a las partes, asumir nuevamente algunos roles que ostentaban tradicionalmente las autoridades ancestrales y que por la práctica diaria y la influencia externa estaban cayendo en desuso.

\section{Conclusiones}

La elaboración del estatuto de Sumubila se realizó a través de un proceso concertado, participativo e incluyente de los diversos actores de la comunidad con el acompañamiento del equipo técnico de la universidad que permitió contar con este instrumento para la gestión comunitaria.

La presente experiencia es una oportunidad para las partes involucradas de trabajar de manera articulada, tomando en cuenta lo estatuido en la política de acompañamiento comunitario intercultural y la guía de articulación institucional, entre otras normativas.

Logramos delimitar los deberes, obligaciones, funciones y mecanismos de articulación entre el equipo técnico de URACCAN y las autoridades comunitarias.

Ello fue posible gracias a la comunicación horizontal y el diálogo abierto entre las partes involucradas, logrando ordenar y cumplir en tiempo y forma las tareas asignadas en pro de un mismo objetivo.

Este proceso contribuyó a la recuperación de memoria colectiva y a un cambio de una práctica oral a un documento escrito, como el caso que nos ocupa. Como dinamizadores del proceso de dialogo de saberes y 
haceres, reconocemos el desafío de escuchar, reflexionar y contextualizar los saberes ancestrales, para la concertación del estatuto comunal debiendo formar parte integral de las normas comunitarias y territoriales al amparo de la ley 28. Estatuto de autonomía; ley 445. Ley de régimen de propiedad comunal y las normativas internacionales que promueven y protegen los derechos de los pueblos indígenas.

Nos permitió transitar por diferentes experiencias en el ámbito comunal - territorial, con una población multiétnica e intercultural como un modo de abrirnos paso a paso en la creación, recreación de conocimientos, saberes y prácticas que fortalecen la estructura tradicional ancestral elaborados con/desde y para la comunidad cumpliendo con uno de sus sueños y aspiraciones en la construcción de un modelo participativo y colaborativo.

\section{Lista de referencias}

Bernal Acevedo, Fabiola (2014). Diálogo de saberes, los aportes de la otredad en la generación de conocimientos. Tesis doctoral, Universidad la Salle, Costa Rica.

Casillas, Lourdes y Laura Santini. (2006). Universidad Intercultural. Modelo educativo, CGEI y B, SEP, México. 287p. citado en González Ortiz, Felipe. La vinculación universitaria en el modelo de educación superior intercultural en México. La experiencia de un proyecto. Ra Ximhai, vol. 7, núm. 3, septiembre-diciembre, 2011, pp. 381-394. Universidad Autónoma Indígena de México. El Fuerte, México.

Mato, Daniel (2009). Contextos, conceptualizaciones y usos de la idea de interculturalidad. En: Miguel Ángel Aguilar y otros, coords. Pensar lo contemporáneo: De la cultura situada a la convergencia tecnológica. Págs.: 28-50. Barcelona y México: Anthropos Editorial y Universidad Autónoma Metropolitana-Iztapalapa.

Mato, Daniel (2016). Educación Superior y Pueblos Indígenas en América Latina: Del "Diálogo de Saberes" a la construcción de "modalidades sostenibles de Colaboración Intercultural". Revista del Cisen Tramas/Maepova, 4 (2), 71-94.

URACCAN (2015). Plan Estratégico Institucional 2015-2019. Managua Nicaragua.

URACCAN (2017). Política de Acompañamiento Comunitario Intercultural. Managua Nicaragua.

URACCAN - IEPA (2018). Ayuda memoria del taller participativo con actores y lideras comunitarios para la recopilación de insumos del estatuto de Sumubila, Tasba Pri, Municipio de Puerto Cabezas. 14 al 17 de mayo del 2018.

URACCAN - IEPA (2018). Borrador del estatuto de Sumubila, Tasba Pri, Municipio de Puerto Cabezas. Octubre 2018.

Copyright ( $($ (URACCAN). Todos los derechos reservados. 